\title{
On the Modeling of Non-Vertical Risers in the Interaction of Electromagnetic Fields With Overhead Lines
}

\author{
Jun Guo ${ }^{\circledR}$, Member, IEEE, Marcos Rubinstein ${ }^{\circledR}$, Fellow, IEEE, Vernon Cooray ${ }^{\circledR}$, Fellow, IEEE, \\ and Farhad Rachidi ${ }^{\circledR}$, Fellow, IEEE
}

\begin{abstract}
This paper proposes a simple method to take into account non-vertical risers through an equivalent partial inductance. The proposed approach was validated considering several examples and taking as reference full-wave results obtained using a numerical electromagnetics code numerical electromagnetics code (NEC)-4 .
\end{abstract}

Index Terms - Induced current, non-vertical riser, total voltage, transient electromagnetic field, transmission line model.

\section{INTRODUCTION}

D UE to difficulties in performing experiments related to lightning-induced voltages on power distribution and transmission lines, engineers depend to a great extent on numerical simulations in making assessments concerning the protection of these systems against lightning.

Several models have been used in the literature to estimate the voltages induced on power lines due to lightning [1]-[7]. While some of these models are actually different representations of the solution to the transmission line equations pertinent to the problem under consideration, the others are either incorrect or partially correct solutions [7]. All these models adhere to the approximation that the response of the overhead transmission line to the incident electromagnetic field is quasi-transverse electromagnetic (TEM) [8].

In the analysis to follow, we will adopt the model presented by Agrawal et al. [1] and we will consider a single overhead conductor, but the conclusions to be extracted from the study are also applicable to all the other models and to multiconductor lines.

\section{Model of AgRaWAl et al.}

Let us consider, without loss of generality, a single-wire line located at a height $h$ above a perfectly-conducting ground plane. The line is located along the $x$-axis and the $z$-axis is aligned with the vertical. The height of the line is $h$. The line impinged upon

Manuscript received December 6, 2018; revised February 18, 2019; accepted February 21, 2019. (Corresponding author: Farhad Rachidi.)

J. Guo and F. Rachidi are with the Electromagnetic Compatibility Group, Swiss Federal Institute of Technology, Lausanne 1015, Switzerland (e-mail: junguo@mail.xjtu.edu.cn; farhad.rachidi@epfl.ch).

M. Rubinstein is with the University of Applied Sciences Western Switzerland, Yverdon-les-Bains 2800, Switzerland (e-mail: marcos.rubinstein@heig -vd.ch).

V. Cooray is with the Department of Engineering Sciences, Uppsala University, Uppsala 75121, Sweden (e-mail: vernon.cooray@angstrom.uu.se).

Color versions of one or more of the figures in this paper are available online at http://ieeexplore.ieee.org.

Digital Object Identifier 10.1109/TEMC.2019.2903335 by an exciting (external) electromagnetic field. We represent the vertical and the horizontal components of the exciting electric field, respectively, by $E_{z}^{e}$ and $E_{x}^{e}$. These exciting electric fields, which are evaluated in the absence of the line conductors, induce currents in the line which, in turn, generate the so-called scattered electric and magnetic fields. Let us define the vertical component of the scattered electric field by $E_{z}^{s}$. The two transmission line equations of the model of Agrawal et al. can be written as

$$
\begin{aligned}
& \frac{\partial v^{s}(x, t)}{\partial x}+R^{\prime} i(x, t)+L^{\prime} \frac{\partial i(x, t)}{\partial t}=E_{x}^{e}(x, 0, h, t) \\
& \frac{\partial i(x, t)}{\partial x}+C^{\prime} \frac{\partial v^{s}(x, t)}{\partial t}=0
\end{aligned}
$$

in which $R^{\prime}, L^{\prime}$, and $C^{\prime}$ are, respectively, the per-unit-length resistance, inductance, and capacitance of the line, $i(x, t)$ is the current, and $v^{s}(x, t)$ is the scattered voltage defined as

$$
v^{s}(x, t)=-\int_{0}^{h} E_{z}^{s}(x, y, z, t) d z .
$$

The total line voltage of the line, $v(x, t)$, can be obtained as

$v(x, t)=v^{s}(x, t)+v^{e}(x, t)=v^{s}(x, t)-\int_{0}^{h} E_{z}^{e}(x, 0, z, t) d z$.

Note that, unlike the scattered electric field in (4), the coordinate $y$ has been set to zero in the exciting field in the integrand on the right hand side of (3) since the exiting field is, in general, not conservative.

The boundary conditions at the two line ends terminated in impedances, $Z_{A}$ and $Z_{B}$, written in terms of the scattered voltages, are given by

$$
\begin{aligned}
& v^{s}(0, t)=-Z_{A} i(0, t)+\int_{0}^{h} E_{z}^{e}(0,0, z, t) d z \\
& v^{s}(L, t)=Z_{B} i(L, t)+\int_{0}^{h} E_{z}^{e}(L, 0, z, t) d z .
\end{aligned}
$$

The equivalent circuit of the Agrawal et al. model equations is shown in Fig. 1. It should be noted that it is implicitly assumed that the risers at the two line ends are vertical and, therefore, the integrals of the vertical electric field in (4)-(6) are performed along the vertical straight line from the ground plane to the conductor. 


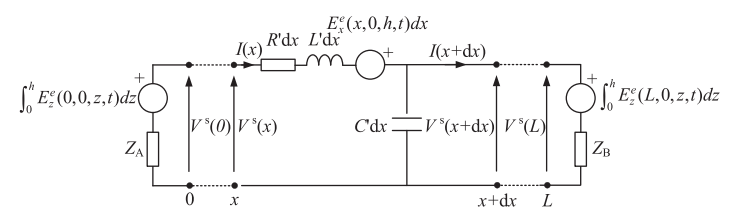

Fig. 1. Equivalent circuit of the Agrawal et al. model for a single-wire line above a perfectly conducting ground plane. The risers at the two line ends are assumed to be vertical in the original model of Agrawal et al.

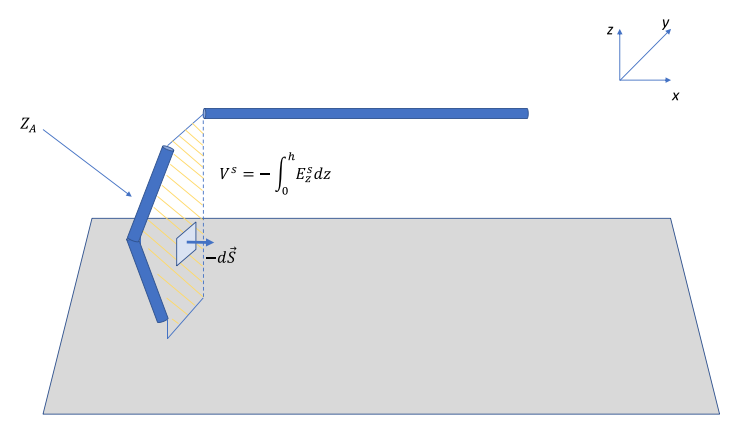

Fig. 2. Non-vertical riser at the left end of the line with a lumped termination impedance.

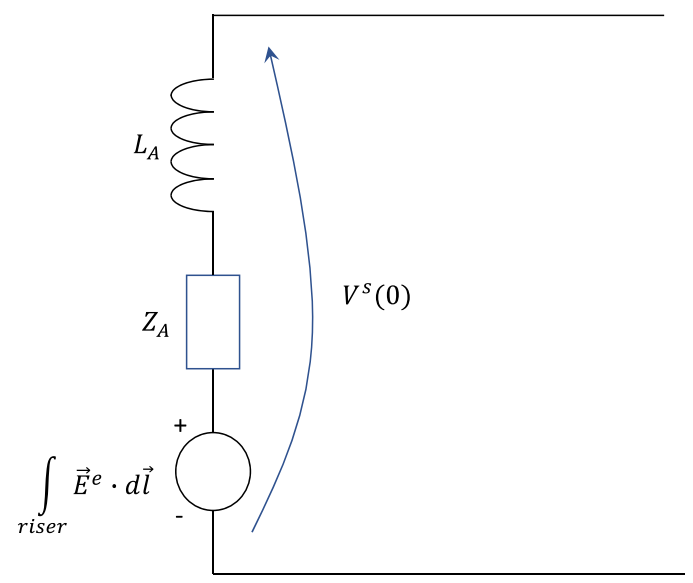

Fig. 3. Equivalent circuit for the left termination when the riser is not vertical.

\section{TREATMENT OF NON-VERTICAL RISERS}

Consider the situation shown in Fig. 2, where the terminal riser is not vertical and is characterized by an arbitrary shape, and not necessarily in the transverse plane.

Unlike the original model of Agrawal et al. since the riser is not vertical, the sources at the terminations are the line integrals of the exiting electric fields along the risers' non-vertical geometries.

Moreover, the termination impedances, represented by $Z_{A}$ and $Z_{B}$ in Fig. 3, are the series combination of the actual termination and an additional inductive impedance stemming from the more general geometry for the risers. In the next section, we present a derivation of these boundary conditions.

\section{A. Boundary Conditions for Non-Vertical Risers}

Integrating along the non-vertical riser on the left-hand side of the line shown in Fig. 2, we can write

$$
-\int_{\text {Riser }} \vec{E} \cdot d \vec{l}=-I(0) Z_{A} \text {. }
$$

Separating the total field into the exiting and scattered components, (7) can be written as follows:

$$
-\int_{\text {Riser }} \vec{E}^{s} \cdot d \vec{l}-\int_{\text {Riser }} \vec{E}^{e} \cdot d \vec{l}=-I(0) Z_{A} .
$$

Now, applying the Maxwell-Faraday's equation to the scattered field around the loop hashed in yellow in the figure, we can write

$$
\int_{\text {Riser }} \vec{E}^{s} \cdot d \vec{l}-\int_{0}^{h} E_{z}^{s} d z=-j \omega \iint \vec{B}^{s} \cdot d \vec{S} .
$$

Solving for the first term on the left-hand side of (9) and substituting the second term by the scattered voltage [see (3)], we obtain

$$
\int_{\text {Riser }} \vec{E}^{s} \cdot d \vec{l}=-V^{s}-j \omega \iint \vec{B}^{s} \cdot d \vec{S} .
$$

Replacing (10) into (8) and solving for the scattered voltage yields

$$
V^{s}=\int_{\text {Riser }} \vec{E}^{e} \cdot d \vec{l}-I(0) Z_{A}-j \omega \iint \vec{B}^{s} \cdot d \vec{S} .
$$

The surface integral in the third term on the right-hand side of (11) is a scattered magnetic flux. Considering the assumption of an electrically small line cross section, this flux is proportional to the termination current, through an inductive term. As a result, we can rewrite (11) as

$$
V^{s}=\int_{\text {Riser }} \vec{E}^{e} \cdot d \vec{l}-I(0) Z_{A}-j \omega L_{A} I(0)
$$

in which the inductance $L_{A}$, whose value depends only on the geometry of the riser, is

$$
L_{A}=\frac{\iint \vec{B}^{s} \cdot d \vec{S}}{I(0)} .
$$

Equation (12) is the result sought. Fig. 3 shows the equivalent circuit for the left-hand side of the line based on (12).

Thus, the integrals of the exciting electric field at the two line ends are evaluated along a path defined by the geometry of the risers.

\section{B. Validation Using Full-Wave Simulations}

In this section, we will use full-wave simulations to validate the equivalent inductance (13) that needs to be considered in the case of non-vertical risers. We will consider a single wire of length $L$, height $h$, and radius $r$ above a perfectly conducting ground. The left and right terminations are, respectively, $Z_{A}$ and $Z_{B}$. The line is illuminated by a uniform plane wave characterized by an azimuth angle $\theta$, an elevation angle $\psi$, and a polarization angle $\alpha$ [see Fig. 4]. The terminal riser at the right end is assumed to be vertical, while three different configurations will be considered for the left-end riser, as shown in Fig. 5(a)-(c).

For the cases of a non-vertical riser [see Fig. 5(b) and (c)], the equivalent (partial) inductance $L_{A}$ should be evaluated using (13). The scattered magnetic field is first evaluated using the Biot-Savart law. Then, the total magnetic flux is computed by numerical integration, taking into account the riser image, as shown in Fig. 6.

The field-to-transmission-line coupling equations including the treatment of the non-vertical risers are solved using the Baum-Liu-Tesche (BLT) equations [9]. According to the BLT 


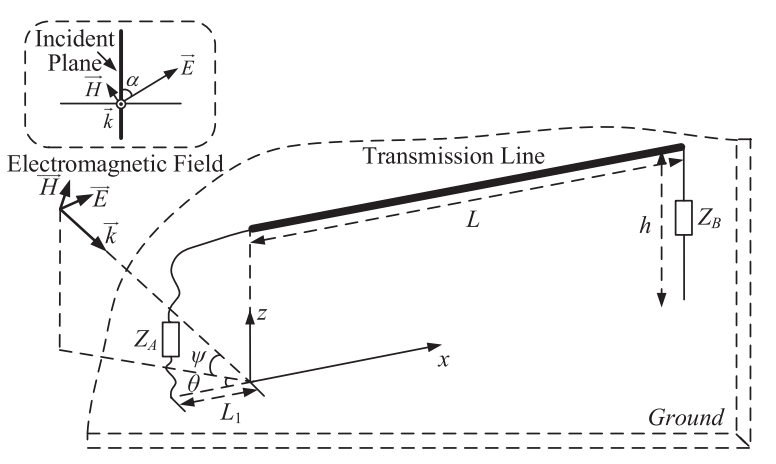

Fig. 4. Single-wire transmission line excited by an incident plane wave.

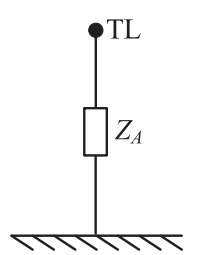

(a)

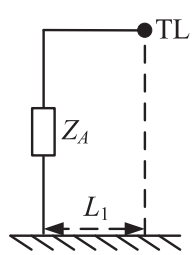

(b)

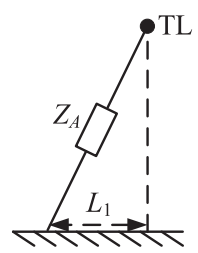

(c)
Fig. 5. Cross-section of the three considered geometries for the left-end riser. (a) Vertical. (b) Rectangular. (c) Triangular.

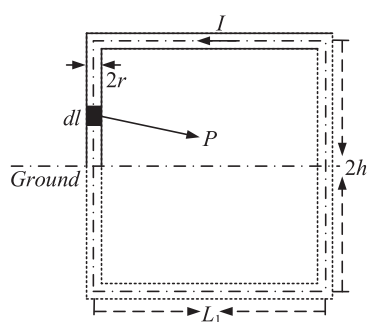

(a)

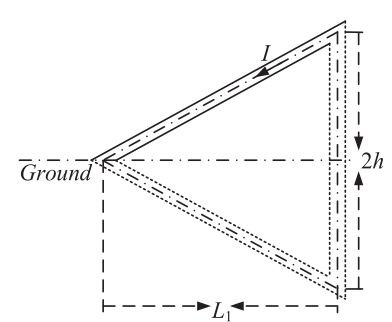

(b)
Fig. 6. Illustration of the geometry for the calculation of the total magnetic flux for the left-end riser. (a) Rectangular. (b) Triangular.

equations, the solution for the induced voltages at both ends can be expressed by

$$
\begin{aligned}
V(0, j \omega) & =\frac{-\rho_{2}\left(1+\rho_{1}\right) S_{1}(j \omega)-e^{\gamma L}\left(1+\rho_{1}\right) S_{2}(j \omega)}{\rho_{1} \rho_{2}-e^{2 \gamma L}} \\
V(L, j \omega) & =\frac{-e^{\gamma L}\left(1+\rho_{2}\right) S_{1}(j \omega)-\rho_{1}\left(1+\rho_{2}\right) S_{2}(j \omega)}{\rho_{1} \rho_{2}-e^{2 \gamma L}}
\end{aligned}
$$

where the source terms $S_{1}$ and $S_{2}$ are given by

$$
\begin{aligned}
S_{1}(j \omega)= & \frac{1}{2} \int_{0}^{L} e^{\gamma x} E_{x}^{e}(x, j \omega) d x-\frac{1}{2} V_{1}(j \omega)+\frac{1}{2} V_{2}(j \omega) e^{\gamma L} \\
S_{2}(j \omega)= & -\frac{1}{2} \int_{0}^{L} e^{\gamma(L-x)} E_{x}^{e}(x, j \omega) d x \\
& +\frac{1}{2} V_{1}(j \omega) e^{\gamma L}-\frac{1}{2} V_{2}(j \omega) \\
V_{1}(j \omega)= & -\int_{\text {Riser } A} \vec{E}^{e} \cdot d \vec{l}, \quad V_{2}(j \omega)=-\int_{\text {Riser B }} \vec{E}^{e} \cdot d \vec{l}
\end{aligned}
$$

in which $\rho_{1}$ and $\rho_{2}$ are the reflection coefficients at the near-end and far-end of the line, respectively, and $\gamma$ is the propagation

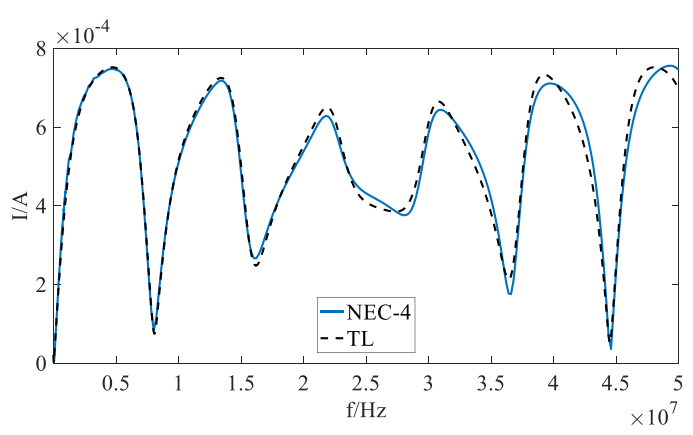

(a)

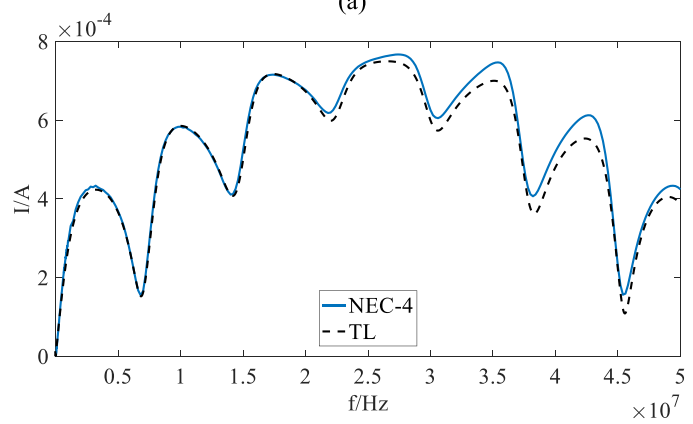

(b)

Fig. 7. Induced currents as a function of frequency. (a) Left end. (b) Right end. The left end of the line is terminated in vertical risers at both ends. The dashed line is used to represent the calculations obtained using the transmission line theory (BLT equations) and the solid line presents the NEC-4 results.

constant along the line. Expressions for the line parameters can be found in [10].

In order to validate the calculation results, the numerical electromagnetics code NEC-4, a full-wave solver based on the method of moments, was used [11]. In what follows, we will consider the three cases for the geometry of the left-end riser shown in Fig. 5.

Case 1: Vertical Risers: In this case, we considered a 20-m long wire located at a height of $0.1 \mathrm{~m}$ above a perfectly conducting ground. The conductor radius is $1 \mathrm{~mm}$. The line is terminated at both ends in $100 \Omega$ resistive loads. The azimuth angle, elevation angle, and polarization angle of the exciting plane wave are $0^{\circ}, 45^{\circ}$, and $0^{\circ}$, respectively. The frequency range of the wave is $10 \mathrm{kHz}-50 \mathrm{MHz}$, and the amplitude of the $E$-field is $1 \mathrm{~V} / \mathrm{m}$ across the whole frequency spectrum. Fig. 7 presents a comparison between the results obtained by way of the BLT equations and of the NEC-4 code for the case of vertical risers. As expected, it can be seen that the results based on the transmission line theory (BLT equations) are in excellent agreement with the full-wave results obtained using the NEC-4.

Case 2: Rectangular Riser at the Left-End: As in Case 1, we considered a $20-\mathrm{m}$ long wire located at a height of $0.1 \mathrm{~m}$ above a perfectly conducting ground and a conductor radius of $1 \mathrm{~mm}$. The line is terminated at both ends in $100 \Omega$ resistive loads. The left-end riser corresponds to Fig. 5(b), while the right-end riser is kept vertical. The azimuth angle, elevation angle, and polarization angle of the exciting plane wave are $0^{\circ}, 45^{\circ}$, and $0^{\circ}$, respectively. The frequency range of the wave is $10 \mathrm{kHz}-$ $50 \mathrm{MHz}$, and the amplitude of the $E$-field is $1 \mathrm{~V} / \mathrm{m}$ across the whole frequency spectrum. We considered two different values for the length $L_{1}$ [see Fig. 5(b)], namely 0.5 and $1 \mathrm{~m}$. For those two lengths, the equivalent inductance $L_{A}$ is, respectively, 0.66 and $1.2 \mu \mathrm{H}$. The calculated results for the induced currents are 


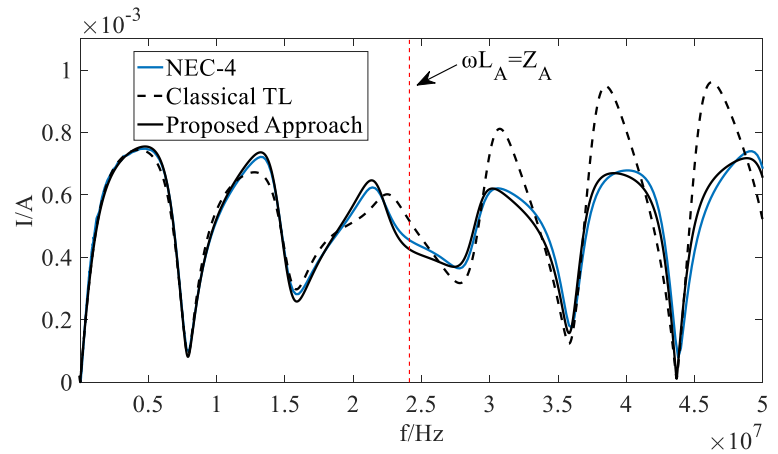

(a)

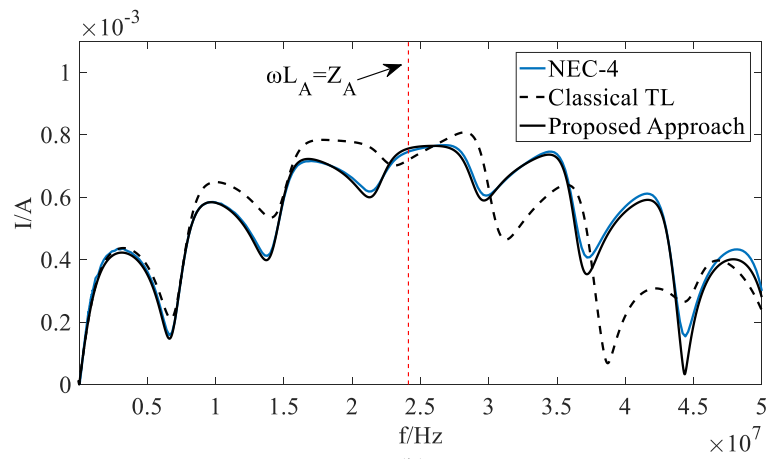

(b)

Fig. 8. Induced currents as a function of frequency. (a) Left end. (b) Right end. The left end of the line is terminated in a rectangular shape riser $\left[L_{1}=0.5 \mathrm{~m}\right.$, Fig. 5(b)], and in a vertical riser on its right end. Calculations obtained using the classical transmission line theory, the transmission line theory including the partial inductance, and the NEC-4 are shown, respectively, using a dashed-line, a solid black line, and a solid blue line.

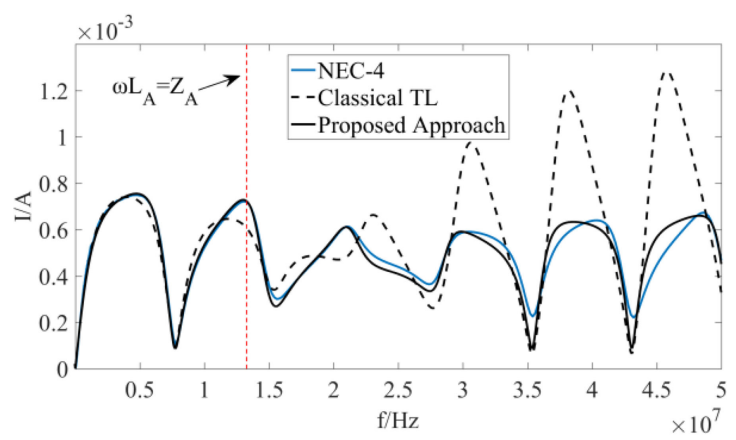

(a)

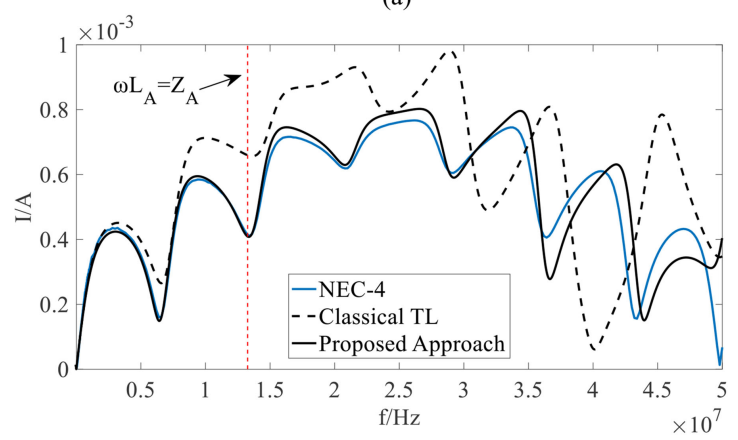

(b)

Fig. 9. Induced currents as a function of frequency. (a) Left end. (b) Right end. The left end of the line is terminated in a rectangular shape riser $\left[L_{1}=1 \mathrm{~m}\right.$, Fig. 5(b)], and in a vertical riser on its right end. Calculations obtained using the classical transmission line theory, the transmission line theory including the partial inductance, and NEC-4 are shown, respectively, using a dashed-line, a solid black line, and a solid blue line.

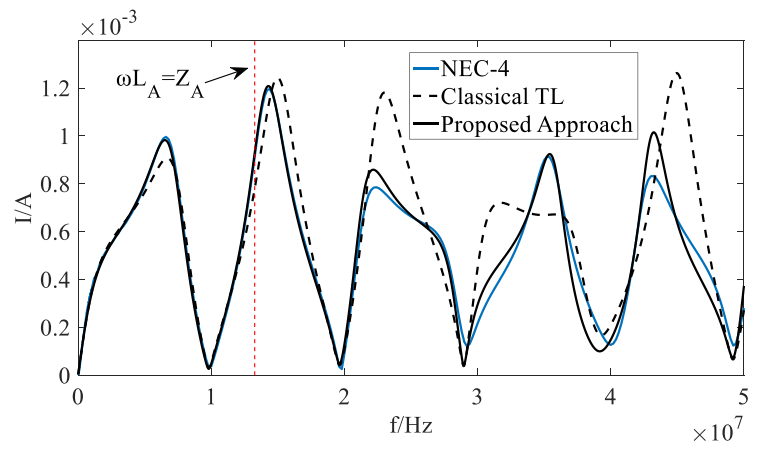

(a)

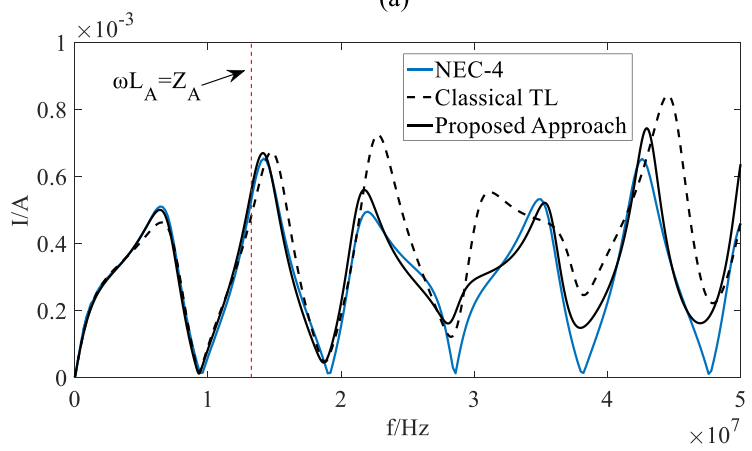

(b)

Fig. 10. Induced currents as a function of frequency. (a) Left end. (b) Right end. The left end of the line is terminated in a rectangular shape riser $\left[L_{1}=\right.$ $1 \mathrm{~m}, \theta=45^{\circ}$, Fig. 5(b)], and in a vertical riser on its right end. Calculations obtained using the classical transmission line theory, the transmission line theory including the partial inductance, and NEC-4 are shown, respectively, using a dashed-line, a solid black line, and a solid blue line.

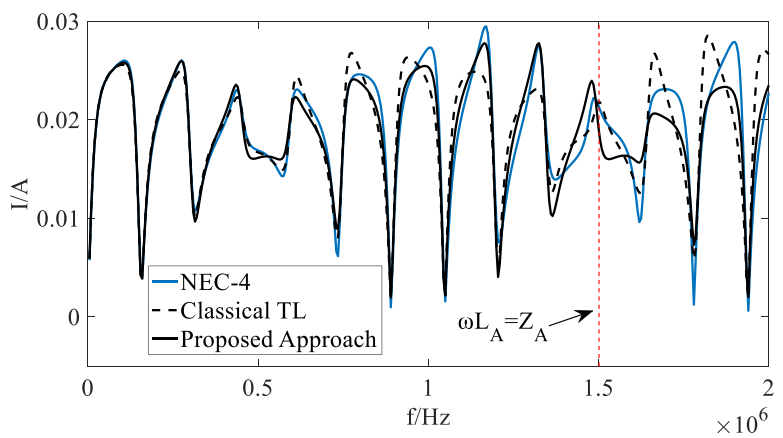

(a)

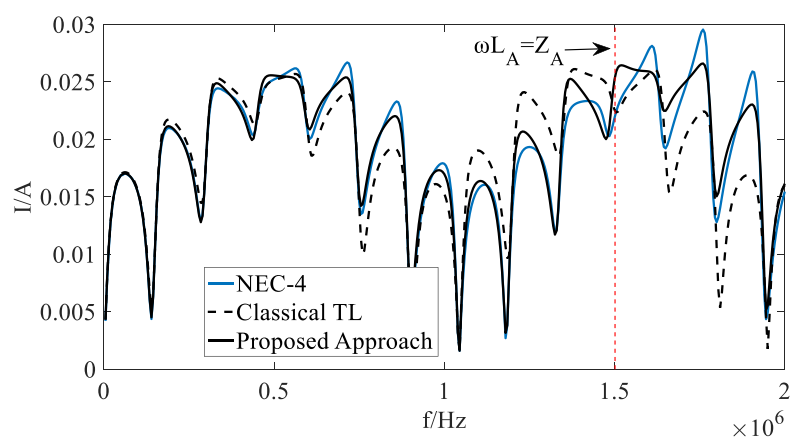

(b)

Fig. 11. Induced currents as a function of frequency. (a) Left end. (b) Right end. The left end of the line is terminated in a rectangular shape riser $[L=$ $1000 \mathrm{~m}, L_{1}=3 \mathrm{~m}$, Fig. 5 (b) ], and in a vertical riser on its right end. Calculations obtained using the classical transmission line theory, the transmission line theory including the partial inductance, and NEC-4 are shown, respectively, using a dashed-line, a solid black line, and a solid blue line. 


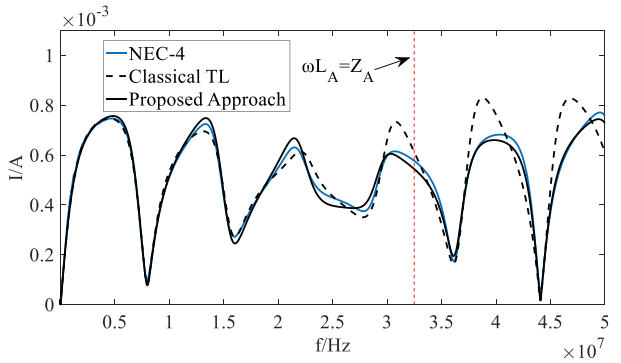

(a)

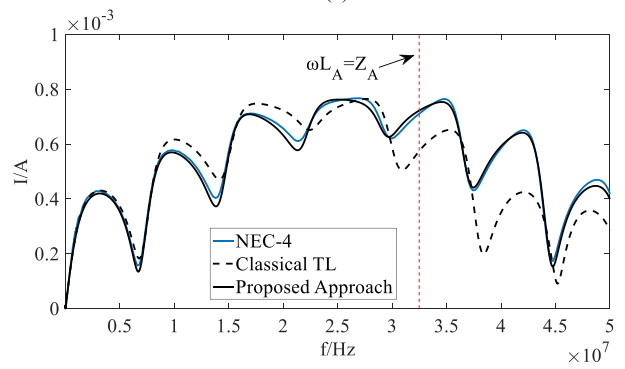

(b)

Fig. 12. Induced currents as a function of frequency. (a) Left end. (b) Right end. The left end of the line is terminated in a triangular shape riser $\left[L_{1}=0.5 \mathrm{~m}\right.$, Fig. 5(c)], and in a vertical riser on its right end. Calculations obtained using the classical transmission line theory, the transmission line theory including the partial inductance, and NEC-4 are shown, respectively, using a dashed-line, a solid black line, and a solid blue line.

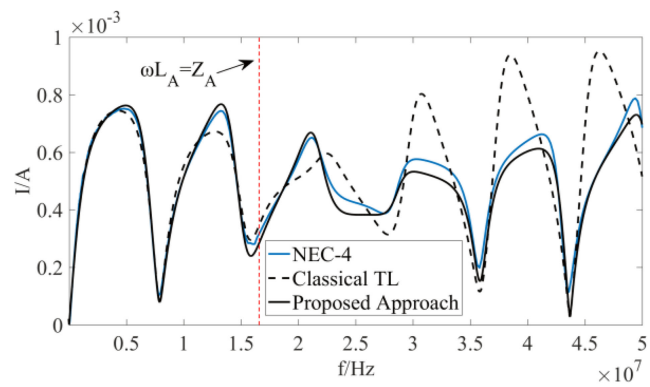

(a)

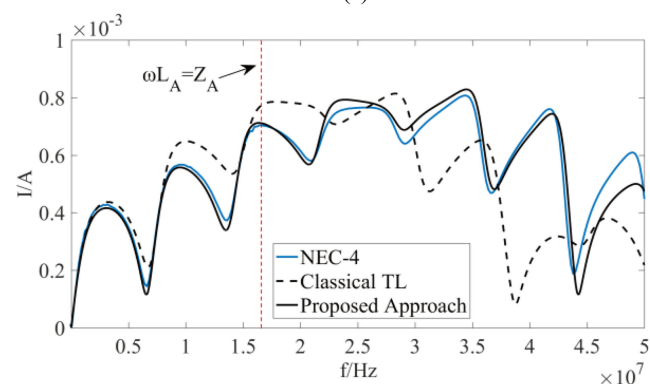

(b)

Fig. 13. Induced currents as a function of frequency. (a) Left end. (b) Right end. The left end of the line is terminated in a triangular shape riser $\left[L_{1}=1 \mathrm{~m}\right.$, Fig. 5(c)], and in a vertical riser on its right end. Calculations obtained using the classical transmission line theory, the transmission line theory including the partial inductance, and NEC-4 are shown, respectively, using a dashed-line, a solid black line, and a solid blue line.

shown in Figs. 8 and 9. It can be seen that the results calculated using the classical transmission line theory deviate from the full-wave results obtained using NEC-4. Taking into account the non-vertical riser using the partial equivalent inductance yields significantly more accurate results.

Note that, in these figures, as well as in the next ones, we have indicated in the plots the frequency at which the impedance of the equivalent inductance becomes equal to that of the terminal impedance. This frequency gives an indication of the frequencies above which this correction needs to be taken into account.

We now consider another example with the same line configuration but considering an azimuth angle of the exciting field of $45^{\circ}$. The calculated results for the induced current are shown in Fig. 10. Again, one can appreciate the fact that the taking into account of the equivalent inductance results in significant improvement of the results, especially at high frequencies.

We also considered the case of a 1000-m long wire at a height of $5 \mathrm{~m}$ above a perfectly conducting ground. The conductor radius is $5 \mathrm{~mm}$. The line is terminated at both ends in $100-\Omega$ resistive loads. The left-end riser corresponds to Fig. 5(b), while the right-end riser is assumed to be vertical. The adopted value for the length $L_{1}$ is $3 \mathrm{~m}$. In this case, the equivalent inductance $L_{A}$ is $10.6 \mu \mathrm{H}$. The azimuth angle, elevation angle, and polarization angle of the exciting plane wave are $0^{\circ}, 45^{\circ}$, and $0^{\circ}$, respectively. The amplitude of the $E$-field is $1 \mathrm{~V} / \mathrm{m}$ across the complete frequency spectrum. The calculated results for the induced current are shown in Fig. 11. Again, it can be seen that the results from the proposed approach agree well with the full-wave results obtained using NEC-4, while the classical transmission line approach fails in reproducing the induced currents in high frequencies.

Case 3: Triangular Riser at the Left-End: In this final case, we considered a $20-\mathrm{m}$ long wire located at a height of $0.1 \mathrm{~m}$ above a perfectly conducting ground. The conductor radius is $1 \mathrm{~mm}$. The line is terminated at both ends in 100- $\Omega$ resistive loads. The left-end riser corresponds to Fig. 5(c), while the right-end riser is kept vertical. We considered two different values for the length $L_{1}$, namely 0.5 and $1 \mathrm{~m}$, respectively. In these two cases, the equivalent inductance $L_{A}$ is respectively 0.49 and $0.96 \mu \mathrm{H}$. The calculated results for the induced currents are shown in Figs. 12 and 13. Again, it can be seen that the proposed approach allows to obtain results that are in an excellent agreement with full-wave results obtained using NEC-4, while the classical transmission line approach fails in reproducing the induced currents, especially in high frequencies.

\section{CONCLUSION}

In this paper, we showed that the classical transmission line theory is not able to accurately take into account non-vertical risers. We proposed a simple method to take into account nonvertical risers through an equivalent partial inductance. The proposed approach was validated considering several examples and taking as a reference full-wave results obtained using NEC-4.

\section{REFERENCES}

[1] A. K. Agrawal, H. J. Price, and S. H. Gurbaxani, "Transient response of a multi-conductor transmission line excited by a nonuniform electromagnetic field," IEEE Trans. Electromagn. Compat., vol. EMC-22, no. 2, pp. 119-129, May 1980.

[2] C. D. Taylor, R. S. Satterwhite, and C. W. Harrison, "The response of a terminated two-wire transmission line excited by a non-uniform electromagnetic field," IEEE Trans. Antennas Propag., vol. AP-13, no. 6, pp. 987-989, Nov. 1965.

[3] F. Rachidi, "Formulation of the field-to-transmission line coupling equations in terms of magnetic excitation fields," IEEE Trans. Electromagn. Compat., vol. 35, no. 3, pp. 404-407, Aug. 1993.

[4] S. Rusck, Induced Lightning Over-Voltages on Power Transmission Lines With Special Reference to the Overvoltage Protection of Low Voltage Networks. Stockholm, Sweden: Elanders Sverige AB, 1958.

[5] P. Chowdhuri, "Analysis of lightning induced voltages on overhead lines," IEEE Trans. Power Del., vol. 6, no. 1, pp. 479-492, Jan. 1989. 
[6] A. Piantini, "Extension of the Rusck model for calculating lightninginduced voltages on overhead lines considering the soil electrical parameters," IEEE Trans. Electromagn. Compat., vol. 59, no. 1, pp. 154-162, Feb. 2017.

[7] V. Cooray, F. Rachidi, and M. Rubinstein, "Formulation of the field-totransmission line coupling equations in terms of scalar and vector potentials," IEEE Trans. Electromagn. Compat., vol. 59, no. 5, pp. 1586-1591, Oct. 2017

[8] F. Rachidi, "A review of field-to-transmission line coupling models with special emphasis to lightning-induced voltages," IEEE Trans. Electromagn. Compat., vol. 54, no. 4, pp. 898-911, Aug. 2012.

[9] C. E. Baum, T. K. Liu, and F. M. Tesche, "On the analysis of general multiconductor transmission line networks," Interact. Note, vol. 350, no. 6, pp. 467-547, 1978.

[10] F. Rachidi, C.A. Nucci, M. Ianoz, and C. Mazzetti, "Influence of a lossy ground on lightning-induced voltages on overhead lines," IEEE Trans. Electromagn. Compat., vol. 38, no. 3, pp. 250-264, Aug. 1996.

[11] J. Burke, Numerical Electromagnetics Code-NEC-4, Method of Moments, Part I: User's Manual and Part II: Program Description-Theory. Livermore, CA, USA: Lawrence Livermore National Laboratory, 1992.

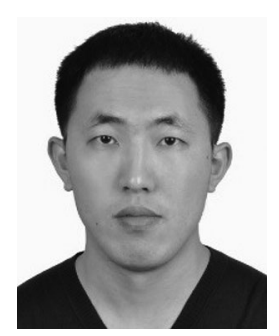

Jun Guo (S'12-M'16) was born in Shanxi, China, in 1986. He received the Ph.D. degree in electrical engineering from Xi' an Jiaotong University, Shaanxi, China, in July 2016.

From 2017 to 2018, he worked as a Postdoctoral with the Electromagnetic Compatibility Laboratory, Swiss Federal Institute of Technology, Lausanne, Switzerland. He is currently working as a Lecturer with the School of Electrical Engineering, Xi' an Jiaotong University. His research interests include transient analysis of multiconductor transmission lines and transient electromagnetic field measurement.

Dr. Guo received several awards including the IEEE Outstanding Young Scientist Award from the 2018 Joint IEEE Electromagnetic Compatibility and Asia-Pacific Electromagnetic Compatibility (APEMC) Symposium, and the International Union of Radio Science (URSI) Young Scientist Award from the 2019 URSI Asia-Pacific Radio Science Conference (AP-RASC).

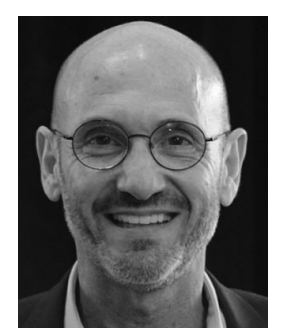

Marcos Rubinstein (M'84-SM'11-F'14) received the master's and Ph.D. degrees, both in electrical engineering, from the University of Florida, Gainesville, FL, USA, in 1986 and 1991, respectively.

In 1992, he joined the Swiss Federal Institute of Technology, Lausanne, Switzerland, where he was involved in the field of electromagnetic compatibility and lightning. In 1995, he was with Swisscom, where he worked in numerical electromagnetics and electromagnetic compatibility. In 2001, he moved to the University of Applied Sciences of Western Switzerland, Yverdon-les-Bains, Switzerland, where he is currently working as a Full Professor, Head of the Advanced Communication Technologies Group, and a Member of the IICT Institute Team. He is the Author or Co-Author of more than 200 scientific publications in reviewed journals and international conferences. He is also the Co-Author of seven book chapters. He is the Chairman of the International Project on Electromagnetic Radiation from Lightning to Tall structures, served as the Editor-in-Chief of the Open Atmospheric Science Journal, and currently serves as an Associate Editor of the IEEE TRANSACTIONS ON ELECTROMAGNETIC COMPATIBILITY.

Prof. Rubinstein received the Best Master's Thesis Award from the University of Florida, Gainesville, FL, USA. He received the IEEE Achievement Award and he is a co-recipient of the NASA's Recognition for Innovative Technological Work Award. He is a Fellow of the IEEE and the SUMMA Foundation, and a member of the Swiss Academy of Sciences and of the International Union of Radio Science.

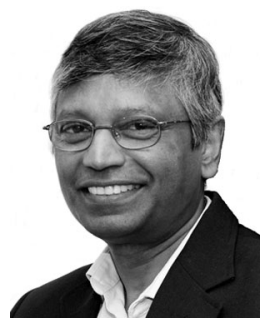

Vernon Cooray ( $\left.F^{\prime} 17\right)$ is currently working as a senior professor at Uppsala University, Uppsala, Sweden. He is responsible for the education and research in atmospheric electrical discharges and lightning. He is also the Professor in Charge of the High Voltage Laboratory, Uppsala University. From 1999-2003, he was the Head of the Division for Electricity.

He has authored and co-authored more than 375 scientific papers. He is the editor of four books, The lightning Flash (2003), Lightning Protection (2009), Lightning Electromagnetics (2012), and The Lightning Flash (second and expanded edition, 2014) published by the Institution of Engineering and Technology (former IEE), London, U. K. He is also the Author of the book, Introduction to Lightning, published by Springer in 2014. For this book, he received the Best Book Award from the Association of Librarians, in 2015.

Prof. Cooray is the President of the ICLP and the recipient of the Berger Award for his contributions to lightning research. In 2015, he was awarded the D.Sc. degree from the University of Colombo, Colombo, Sri Lanka, for his contributions to lightning research.

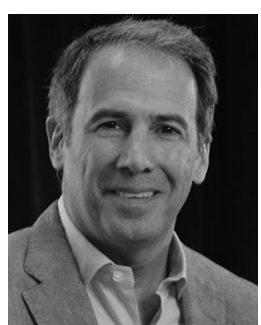

Farhad Rachidi (M'93-SM'02-F'10) received the M.S. degree in electrical engineering and the Ph.D. degree from the Swiss Federal Institute of Technology, Lausanne, Switzerland, in 1986 and 1991, respectively.

He was with the Power Systems Laboratory, Swiss Federal Institute of Technology, until 1996. In 1997, he joined the Lightning Research Laboratory, University of Toronto, Toronto, ON, Canada. From 1998 to 1999 , he was with the Montena EMC, Rossens, Switzerland. He is currently working as a Titular Professor and the Head of the Electromagnetic Compatibility Laboratory, Swiss Federal Institute of Technology. He has authored or co-authored more than 190 scientific papers published in peer-reviewed journals and more than 400 papers presented at international conferences.

Dr. Rachidi is currently a member of the Advisory Board of the IEEE TRANSaCtions ON ElECtromagnetic COMPATIBILITY and the President of the Swiss National Committee of the International Union of Radio Science. He has received numerous awards including the 2005 IEEE EMC Technical Achievement Award, the 2005 CIGRE Technical Committee Award, the 2006 Blondel Medal from the French Association of Electrical Engineering, Electronics, Information Technology and Communication (SEE), the 2016 Berger Award from the International Conference on Lightning Protection, the 2016 Best Paper Award of the IEEE TRANSACTIONS ON ELECTROMAGNETIC COMPATIBILITY, and the 2017 Motohisa Kanda Award for the most cited paper of the IEEE TRANSACTIONS ON ELECTROMAGNETIC COMPATIBILITY (2012-2016). In 2014, he was conferred the title of Honorary Professor of the Xi' an Jiaotong University, Shaanxi, China. He served as the Vice-Chair of the European COST Action on the physics of lightning flash and its effects from 2005 to 2009, the Chairman of the 2008 European Electromagnetics International Symposium, the President of the International Conference on Lightning Protection from 2008 to 2014, the Editor-in-Chief of the Open Atmospheric Science Journal (2010-2012), and the Editor-in-Chief of the IEEE TRANSACTIONS ON ELECTROMAGNETIC COMPATIBILITY from 2013 to 2015. He is a Fellow of the IEEE and of the SUMMA Foundation, and a member of the Swiss Academy of Sciences. 